\title{
Driving backwards using a semi-autonomous smart wheelchair system (DSS): A clinical evaluation
}

\author{
Vinod Sharma ${ }^{\mathrm{a}, \mathrm{b}, *}$, Richard Simpson $^{\mathrm{a}, \mathrm{b}, \mathrm{c}}$, Edmund LoPresti $^{\mathrm{d}}$ and Mark Schmeler ${ }^{\mathrm{b}}$ \\ ${ }^{a}$ Department of Bioengineering, University of Pittsburgh, Pittsburgh, PA, USA \\ ${ }^{\mathrm{b}}$ Department of Rehabilitation Science and Technology, University of Pittsburgh, Pittsburgh, PA, USA \\ ${ }^{\mathrm{c}}$ Human Engineering Research Labsoratory, VA Pittsburgh Healthcare System, Pittsburgh, PA, USA \\ d AT Sciences, Pittsburgh, PA, USA
}

\begin{abstract}
Some wheelchair users have difficulty looking backward when backing up in confined spaces due to limited neck range of motion or low vision, which can lead to collisions which may result in personal injury or property damage. The Drive Safe System (DSS) was evaluated in a controlled laboratory setting with blindfolded able-bodied individuals on various backward driving tasks. Performance with the DSS was compared with a standard white cane used for navigation assistance by people with visual impairment. Results indicate that the DSS significantly reduced the number of collisions compared to using a cane $(p=0.0001)$ alone. There was no difference in task completion time when participants were using the cane or the DSS $(p=0.915)$. Users rated the DSS favourably as they experience less total workload $(p=0.026)$, less physical demand $(p=0.006)$, felt less frustrated $(p=0.002)$ and put less effort $(p=0.007)$ to achieve better performance when using the DSS, compared to using a cane. These findings suggest that the DSS can be a viable powered mobility solution for wheelchair users with visual impairments.
\end{abstract}

Keywords: Intelligent mobility aids (IMAs), collision avoidance, smart wheelchair, robotics, embedded distributed system, semi-autonomous system

\section{Introduction}

\subsection{Problem statement}

Independent mobility is a key component in maintaining the physical and psychosocial health of an

*Corresponding author: Vinod Sharma, Ph.D., ARRT Postdoctoral Fellow, Department of Rehabilitation Science and Technology, University of Pittsburgh, Bakery Square 853, 6465 Penn Avenue, Suite 401, Pittsburgh, PA 15206, USA. Tel.: +1 4129532157 (M); Fax:+1 412624 6501; E-mails: vks3@pitt.edu (V. Sharma), ris20@ pitt.edu (R. Simpson), edlopresti@at-sciences.com (E. LoPresti), schmeler@pitt.edu (M. Schmeler). individual [1-3]. Psychologically, a decrease in mobility can lead to feelings of emotional loss, anxiety, depression, reduced self esteem, social isolation, stress, and fear of abandonment. Reduction in functional mobility is linked with reduced participation and loss of social connections [2]. Further, for people with disabilities, independent mobility increases vocational and educational opportunities, reduces one's dependence on caregivers and family members, and promotes feelings of self-reliance [3]. Many non-institutionalized individuals with mobility impairments have been shown to improve their performance and satisfaction in activities of daily living (ADL) by using a powered wheelchair [1, 4-7]. 
Powered wheelchairs offer the benefits of independent mobility while allowing individuals to devote their energy towards ADL $[8,9]$. Many ADL such as toileting, dressing, feeding, transferring, getting in and out of public transportation, getting in and out of elevators, and ascending or descending ramps require a person to manoeuvre a wheelchair in confined spaces. In order to access these confined spaces, drivers may be required to drive in reverse or perform manoeuvres similar to parallel parking. Many wheelchair users have limited neck range of motion and have difficulty looking backward when backing up in confined spaces. This creates unsafe driving practices and can lead to collisions which may result in personal injury or property damage.

A powered wheelchair data logger study [10] showed that, for non-institutionalized powered wheelchair users, $13 \%$ of their total travel distance is driven backwards. Distance travelled between stops when moving forward was 11.65 meters and 2.73 meters when driving backwards. Speed of travel forward and backward for this population was not significantly different. Driving backward is difficult because of poor reverse directional stability of powered wheelchairs [11]. Caster orientation and driving speed can vary the reverse movement direction of the powered wheelchair, requiring multiple joystick manoeuvres to achieve the desired movement direction [11].

Even though the benefits of powered mobility are well documented, the safety issues associated with operation of powered wheelchairs often prevents clinicians and rehab practitioners from prescribing powered mobility [12-14]. Many researchers have investigated the wheelchair related incidents and injuries in institutional and non-institutional wheelchair users [13-15]. A survey of 109 wheelchair users reported 253 incidents occurring within a 5-year period, $27 \%$ of which required medical visits and $19 \%$ of which required hospitalizations [13]. The majority of these incidents and injuries involved power wheelchairs. One obstacle to operating a powered wheelchair safely is impaired vision. The American Federation for the Blind (AFB) has estimated that $9.61 \%$ of all individuals who are legally blind also use a wheelchair or scooter, in addition to another $5.25 \%$ of individuals who have serious difficulties seeing, but are not legally blind [16]. Further, $5.3 \%$ of all wheeled mobility equipment (WME) users are either legally blind or have serious difficulty in seeing [17].
Visual and physical impairments often accompany the natural aging process. Macular degeneration, cataracts, glaucoma, and diabetic retinopathy are the leading causes of visual impairments among older adults. According to the 2007 disability status report [18], $40.3 \%$ of non-institutionalized individuals age 75 and older in the US have conditions that substantially limit one or more basic physical activities such as walking, climbing stairs, reaching, lifting, or carrying. Further, $23.6 \%$ of individuals in this population have sensory disabilities, which include blindness or severe visual impairment. The percentage of wheelchair users who are age 65 or above has steadily increased from $2.74 \%$ in 1990 to $5.2 \%$ in 2005. $12.28 \%$ of the non-institutionalized population age 85 and over uses wheelchairs [17], most of which are manual wheelchairs pushed by a caregiver or a family member.

There is limited literature addressing the use of powered wheelchairs by individuals with combined visual and mobility impairments. A case study of a visually impaired, powered wheelchair user who uses a white cane for navigation assistance was presented in [19]. Another case study of the use of a guide dog with a powered wheelchair by a visually impaired person is presented in [20]. Other authors [21,22] have evaluated the merits and limitations of using a white cane with manual and power wheelchairs. Some researchers also advocate the use of power assisted manual wheelchairs for this population [16, 23].

To accommodate people who are not served by existing wheelchair technology, several researchers have used mobile robotics technologies to create "smart wheelchairs". A smart wheelchair typically consists of either a standard power wheelchair to which a computer and a collection of sensors have been added or a mobile robot base to which a seat has been attached. The use of smart wheelchairs has been researched since the early 1980s as a form of assistance for people who lack the visual, motor, or cognitive skills required to drive a powered wheelchair [24]. A detailed discussion of the population of users who could benefit from smart wheelchairs was provided in [6]. Despite a long history of research in smart powered wheelchairs, very few smart wheelchairs are available commercially [24, 25]. The Wheelchair Pathfinder (a commercial product sold by Nurion Industries that has been discontinued) [25], was an electronic mobility aid which detected potential obstacles and alerted the user to their presence, to assist users who are blind or have low vision 
or who have limited arm or head control. The CALL Center smart powered wheelchair is sold in Europe by Smile Rehab, Ltd. (Berkshire, UK) and includes bump sensors, sonar sensors and the ability to follow tape tracks on the floor [26].

Smart wheelchair technologies have shown considerable advantages in reducing the number of collisions in comparison to conventional navigation assistance methods. However, no study has yet evaluated a person's performance on a navigation task which requires driving in reverse. This is an important consideration, because people driving backwards are more prone to collisions due to poor reverse directional stability of power wheelchairs that vary with the castor orientation, driving speed, and the centre of gravity of the wheelchair.

\subsection{The Drive Safe System (DSS)}

The Drive Safe System (DSS) builds upon our prior work on the development of an economically sustainable smart wheelchair system which provides collision avoidance at a low cost and with minimal modifi- cations to the underlying wheelchair or environment $[27,28]$. In this project, the DSS was redesigned from earlier prototypes to achieve a more robust, commercially viable design. The DSS is an add-on, distributed, shared control navigation assistance system for powered wheelchairs, intended to provide safe and independent mobility (Fig. 1a). The user is responsible for high-level control of the system, such as choosing the destination, path planning, and some navigation actions, while the DSS overrides unsafe manoeuvres through autonomous collision avoidance, automatic wall following, and door crossing. In this project, the DSS was redesigned from earlier prototypes to achieve a more robust, commercially viable design. The resulting system was tested for performance goals such as sensor coverage, maximum safe speed, maximum detection distance, and power consumption.

The DSS has a modular architecture, with a central control and interface node (the Joystick Translator) communicating with one or more Sensor Nodes (Fig. 2). The Sensor Nodes monitor the environment for potential obstacles and deliver auditory and visual alerts to the user. Each sensor node consists of five

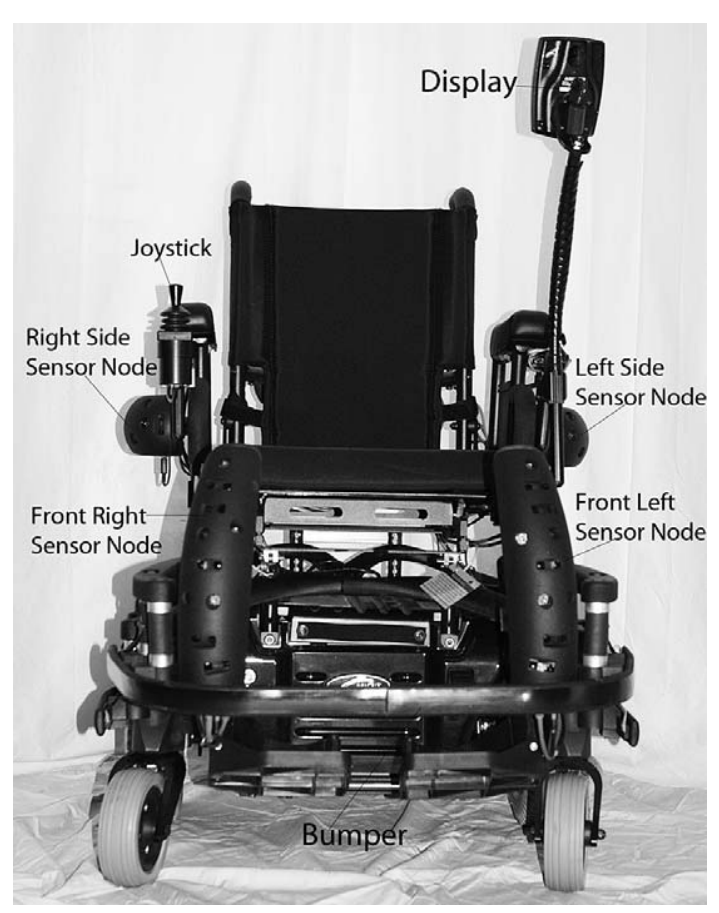

(a)

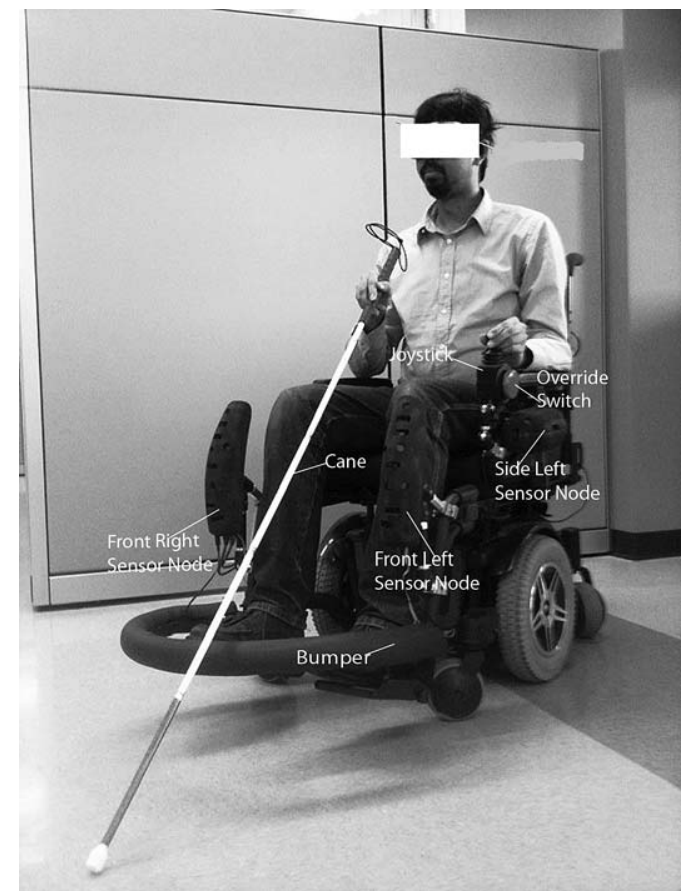

(b)

Fig. 1. (a) The Drive Safe System (DSS). (b) Blindfolded able bodied individual using the DSS with a cane. 


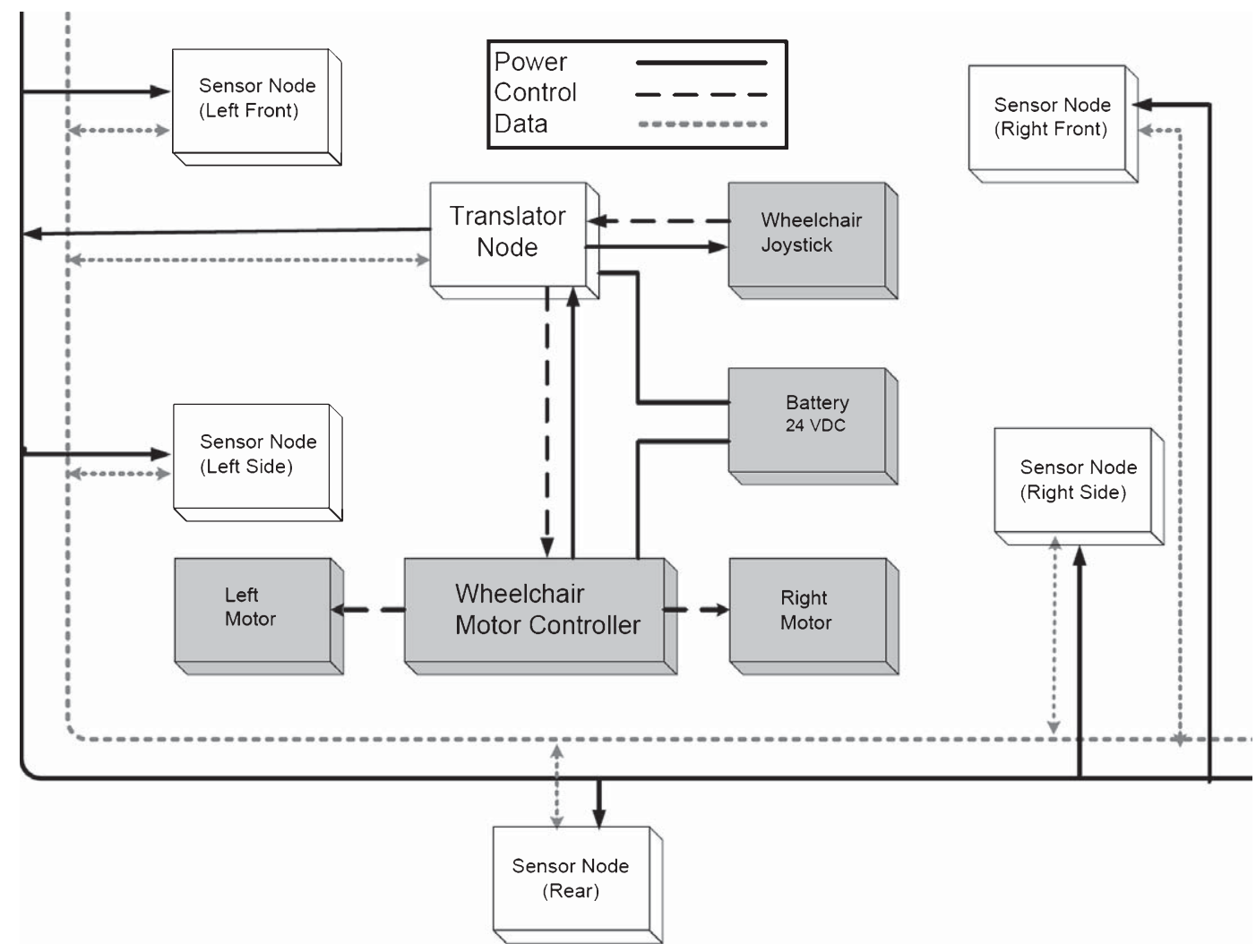

Fig. 2. The DSS architecture.

ultrasonic rangefinders (URs), five infrared rangefinders (IRs), two touch sensitive bumpers, one speaker, and three status LEDs. The DSS uses a Controller Area Network (CAN 2.0) for communication between nodes. The DSS CAN bus operates at 1 Megabits Per Second (MBPS) and uses 29-bit extended identifier messages for communication.

The DSS implements five operating modes: Obstacle avoidance mode, door crossing mode, wall following mode, corridor crossing mode, and override mode. The DSS software is distributed across the sensor nodes and the translator node but the translator node plays the central role in implementing various modes and behaviours. The Translator node's function is to intercept the user's joystick signals and send a modified joystick signal to the wheelchair motor controller. The Translator maintains the current state of each sensor node element (e.g. sonar range information, IR range information, bumper state, state of the speaker and LEDs) based on data from the sensor nodes. Sensor data is used to check for the presence of obstacles in the direction the user is pointing the joystick.
Typically, the Translator provides a signal to the motor controller which is identical to the original joystick signal, preserving the user's control. However, if the system detects a collision risk, the Translator will scale the signal (slowing the wheelchair) or send a neutral signal (stopping the wheelchair). Many researchers $[29,30]$ have advocated user adaptive shared control architecture in which both speed and direction of the wheelchair can be altered by the underlying system to maintain the safety of individuals while providing a high degree of autonomy. The DSS on the other hand, does not vary the direction of travel from the user's original intent, so that the user feels more in control of the wheelchair, and can steer the wheelchair away from obstacles themselves rather than the wheelchair steering them away from the obstacle. Not changing the intended direction enables users to perform ADLs (e.g. reaching a faucet, reaching water fountain, reaching desk, and reaching towards toilet seat) without getting frustrated if the system keeps driving them away from what it considers obstacles (e.g. faucet, water fountain, desk, and toilet seat). 
The specific behaviour of the system depends on the operating mode. A detailed description of the DSS hardware and software is being published elsewhere [31]. The DSS divides the area around the wheelchair into 16 angular sectors (each 22.5 degrees) and each of these sectors relates to a possible direction of movement of the wheelchair based on the joystick position. The translator node gathers information from all the sensor nodes and organizes this information in an obstacle density map (ODM) database which shows the position of obstacles in these 16 sectors. The translator samples the joystick at $20 \mathrm{~Hz}$, and the joystick signal is analyzed to determine the sector where the driver intends to move. Based on the intended direction of movement and obstacle density from the ODM in that sector, the translator can make three choices:

- First, no change in the input speed and direction signals, if there are no obstacles in intended movement direction within $255 \mathrm{~cm}$.

- Second, slow down the wheelchair if the nearest obstacle in the path of travel is between 255 and $35 \mathrm{~cm}$ (slow down region) away.

- Third, stop the wheelchair if the nearest obstacle in the path of travel is less than $35 \mathrm{~cm}$ (stop region) away.

The touch sensitive bumper modules provide additional protection in case the UR and IR sensors fail to detect an obstacle. When a bumper segment is pressed, the DSS will not allow movement in the sectors covered by the pressed bumper and pushes the wheelchair away from the obstacle with a spring reaction. Whenever the translator stops the wheelchair because of an obstacle, users are notified through auditory and visual feedback. The user has the option to steer away from the obstacle in the direction of any clear sector, allowing the user to manoeuvre around the obstacle and continue moving. The DSS provides auditory feedback when the wheelchair is stopped by the DSS in the presence of an obstacle, whenever the bumpers are touched by an obstacle, and whenever the driver operates the wheelchair in override mode.

The DSS is designed to act as a specialty user interface, allowing it to connect with the control electronics of a variety of wheelchairs. The current DSS prototype has been tested on wheelchairs from Pride Mobility (www.pridemobility.com) and Sunrise Medical (www.sunrisemedical.com), and past prototypes were tested on wheelchairs by Invacare (www.invacare.com), Permobil (www.permobil.com), and Everest and Jennings (www.grahamfield.com) [28]. The wheelchair joystick (or other control device) plugs into the Joystick Translator, and the Joystick Translator plugs into the wheelchair's motor controller. The Joystick Translator also obtains power from the wheelchair batteries, and provides power to the Sensor Nodes.

The DSS can be used in areas that have been modified to (1) reduce the likelihood of sensor failure and (2) limit the consequences of sensor failure. We believe that an individual who is motivated to use the DSS will be willing to make simple modifications to the environment(s) in which the DSS will be used. These modifications include (1) eliminating or obstructing glass walls and doors, (2) moving valuable, breakable things to places where the wheelchair cannot break them, (3) using baby gates or doors to block stairwells, and (4) widening doorways to at least 32 inches. The DSS can be used as a regular wheelchair in unmodified environments, but cannot be relied on as a smart wheelchair.

\section{Method}

\subsection{Subjects}

This study employed able-bodied individuals wearing blindfolds to simulate complete blindness. Using able-bodied subjects made it possible to recruit a large number of homogeneous participants, which facilitated group statistical analyses. However, the able-bodied participants were not experienced wheelchair users and did not have the orientation and mobility skills of people with visual impairments. The study protocol was approved by the Institutional Review Board (IRB) of the University of Pittsburgh. Nineteen participants (6 females, 13 males) were recruited for this study. The mean age of participants was 28.4 years (SD 3.9 y). Three participants had prior experience with power wheelchair driving but none was a regular wheelchair user. The subjects who participated in this study also participated in the forward driving study with the DSS in [32].

\subsection{Apparatus}

A Quantum-600 mid-wheel drive powered wheelchair (manufactured by Pride Mobility Products) was 
equipped with the DSS (Fig. 1b) for this study. The wheelchair was without tilt, recline and seat elevation functions and its seat width was $45 \mathrm{~cm}$. The wheelchair was controlled by a proportional joystick. The maximum forward speed of the wheelchair was set to $75 \mathrm{~cm} / \mathrm{sec}$ and the maximum reverse speed was set to $58 \mathrm{~cm} / \mathrm{sec}$ to closely match the average driving speed for wheelchair users [33]. An area of $3.30 \times 7.50$ sq. meters was used for the user trial. Safety glasses covered with paper tape on the front and sides were used as a blindfold. Cylindrical cardboard tubes $20 \mathrm{~cm}$ diameter and $152 \mathrm{~cm}$ tall were used as obstacles, and wooden benches $91.44 \mathrm{~cm}$ height were used to mark the boundary of the area.

\subsection{Procedure}

\subsubsection{Pre-training}

Prior to participating in the study, each participant read the Informed Consent Form. Once each participant indicated that the form had been read and understood, and agreed to participate, the informed consent form was signed. A copy of the informed consent form was given to each participant upon completion of the experiment.

Depending upon each participant's requirements, the seating and positioning of the wheelchair was adjusted by the investigator. For example, the wheelchair joystick was mounted on the right or the left side of the wheelchair, depending upon whether the participant was left- or right-handed. Backrest and footrests were adjusted to allow participants to sit comfortably in the wheelchair. Once participants were comfortable with their seating and the positioning of the joystick, they were introduced to the wheelchair controller and the joystick interface. The experimenter explained the functioning of the wheelchair, the manoeuvring of the wheelchair using a proportional joystick, and controller parameters such as maximum forward speed, maximum reverse speed, acceleration, and deceleration.

\subsubsection{Training}

Participants were required to demonstrate verbal understanding of the system before they were given training on how to manoeuvre the wheelchair using the joystick. Participants' wheelchair driving skills were tested on two courses designed to enhance participants' familiarity with the wheelchair's dynamics and ability to manoeuvre in tight spaces without a blindfold. Each participant traversed these courses while driving forward and then backwards until the participant was able to traverse the courses without hitting any obstacles. While driving on the test course, participants did not have support from the DSS UR and IR sensors, but the touch sensitive bumpers were active; if a participant hit an obstacle the bumpers would stop the wheelchair.

Next, participants learned to drive the wheelchair with a cane while blindfolded (see Fig. 1b). Participants used a 48 in $(121.92 \mathrm{~cm})$ tall white cane to scan for obstacles in the environment. Participants were given instructions on how to use the cane to scan the environment and detect obstacles while moving forward and while moving backwards. Participants used their dominant hand to operate the joystick and their non-dominant hand to scan the environment with the cane. During training phase participants were asked to complete two obstacle courses while driving backwards to practice navigation while blindfolded and using a cane.

Once participants felt comfortable and confident with using the cane, they received training on operating the wheelchair with the DSS. Participants received an explanation about the DSS architecture, its various behaviours and the logic the DSS uses to avoid collisions. Participants received an explanation about the auditory feedback patterns generated by the DSS when there is an obstacle in the direction of travel. As part of the training, participants were blindfolded and asked to localize the position of the obstacles based on auditory feedback from the DSS. When participants stated that they understood the DSS and its operation, they were asked to approach obstacles placed in front of the wheelchair to observe the wheelchair's response to obstacles. Participants were then asked to approach obstacles placed to the side and rear of the wheelchair to observe the DSS's response in these situations. Participants were then blindfolded and asked to complete two obstacle courses using assistance from the DSS. Investigators observed the performance of the participants and instructed them on various navigation skills to use the assistance from the DSS effectively. These training courses gave participants an understanding of the obstacle distance thresholds of the DSS in various directions around the wheelchair.

The last set of training activities involved the use of the cane and the DSS together. Participants were instructed to use the cane to determine the location 
of obstacles when the DSS stopped the wheelchair. Participants were instructed to hold the cane on their lap or in a position where it did not interfere with the UR and IR sensors when it was not being used. Participants were asked to complete two training obstacle courses in this condition to familiarize themselves with the use of the cane with the DSS.

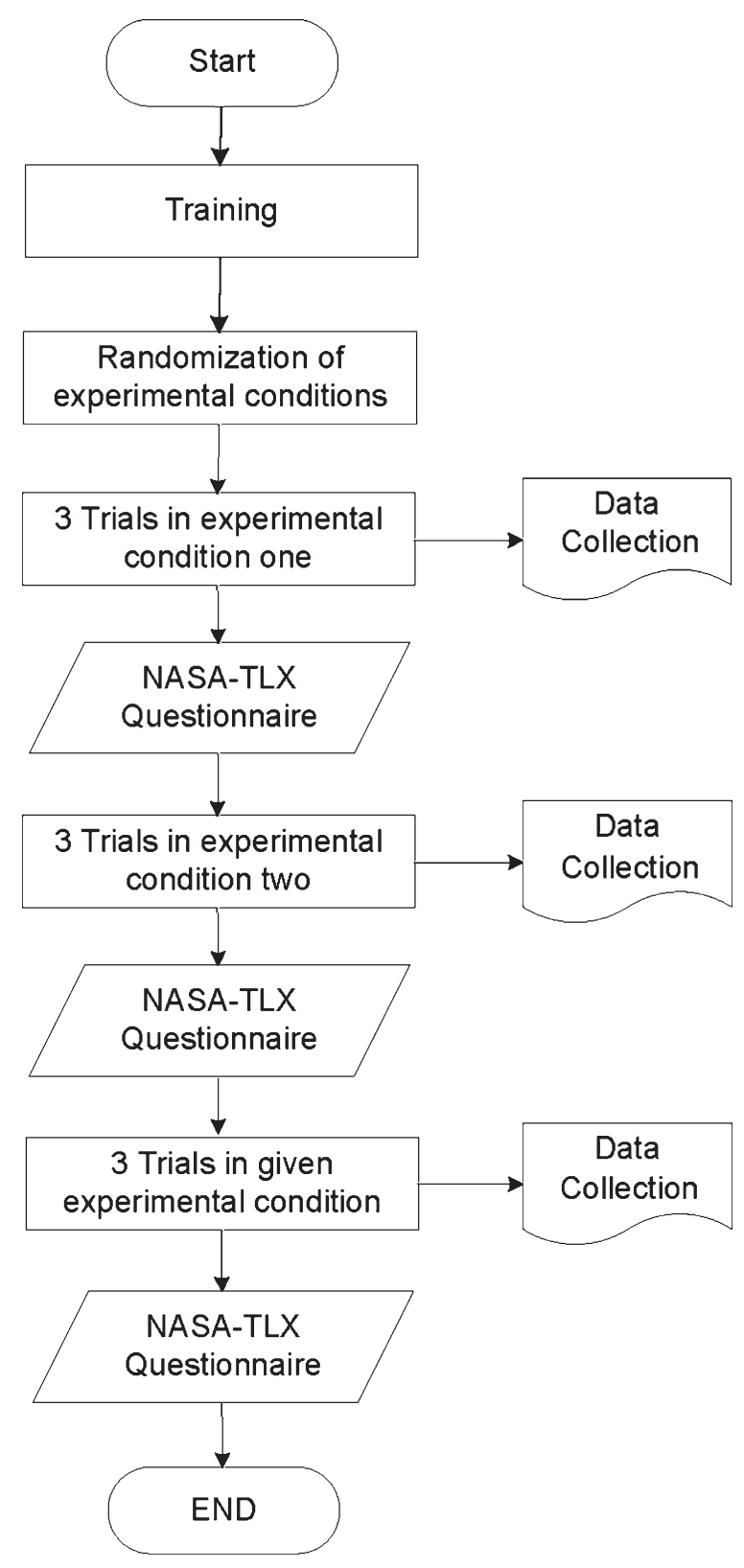

Fig. 3. Experimental protocol.

\subsubsection{Experiment protocol}

Protocol for this study is shown in Fig. 3. Participants completed three trials under each of three experimental conditions:

(1) Cane: Participant used a $48^{\prime \prime}$ white cane for navigation assistance while driving the powered wheelchair

(2) DSS: Participant used the DSS for navigation assistance.

(3) Cane\&DSS: Participant used both the cane and the DSS for navigation assistance. Participants were instructed to use the DSS when the wheelchair was moving, and to use the cane to find the position of obstacles when the wheelchair was stopped by the DSS.

In each trial, participants were blindfolded and asked to reach a goal indicated by a sound source (portable radio or laptop computer providing continuous music) while driving the wheelchair backwards. The order of experimental conditions (Cane, DSS, and Cane\&DSS) and obstacle courses (see Fig. 4) was randomized. Participants were given four minutes to complete each trial while driving backwards. If participants were unable to finish the trial within four minutes, they were asked to restart the trial and the obstacle course in the retrial was different. One investigator carried a data collection sheet and filled in the sheet with observations about time and collisions during each trial. The other investigator walked behind the wheelchair and could bring the wheelchair to an immediate halt if a risk of danger to the participant was perceived. If participants displaced an obstacle from its location, the obstacle remained in its displaced location until the end of the trial. If a participant knocked over an obstacle, investigators removed the obstacle from the wheelchair's immediate path of travel. Once the bumper in the backside of the wheelchair crossed the finish line participants were told to bring the wheelchair to a stop. The participant remained blindfolded while being moved back to the starting position for the next trial and the obstacle course was changed. After finishing the three trials in each condition, participants completed the NASATLX questionnaire (Hart, 2006, 35).

2.3.3.1. Dependent variables. Task Completion Time (TCT): TCT was the time taken by a participant to navigate the obstacle course from the starting position to the finish line, measured in seconds. 

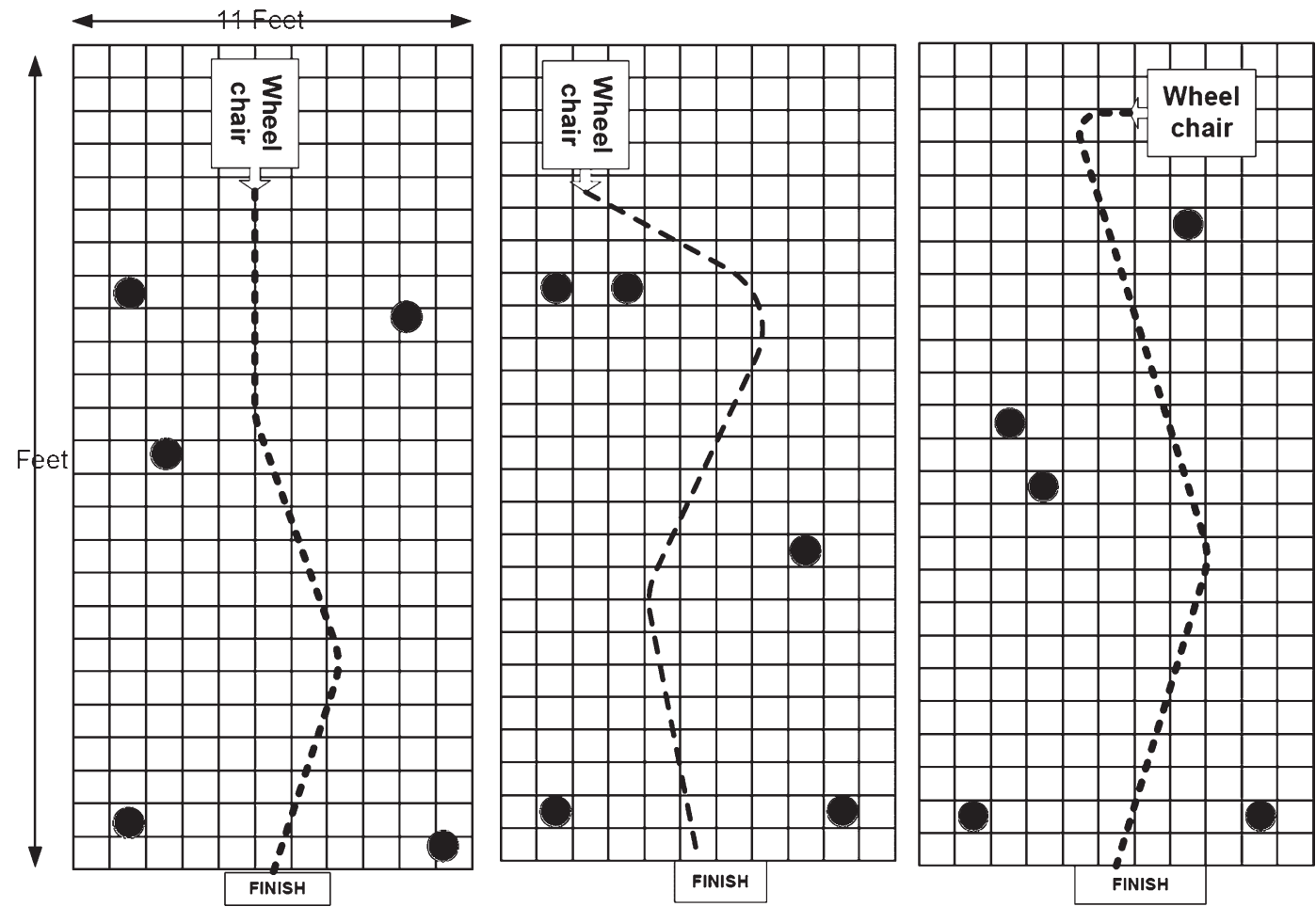

Fig. 4. Obstacle Courses

Total Number of Collisions (TNC): A collision was defined as contact between the wheelchair and a cardboard tube or boundary wall. TNC quantifies the total number of collisions across all three trials in each experimental condition (Cane, DSS, and Cane\&DSS). The intensity and severity of a collision was determined based on the displacement of an obstacle from its initial position (Fig. 5a):

Type I collision: Collisions in which a cardboard tube was displaced by less than two inches (Fig. 5b) or the wheelchair touched the surrounding wall without displacing the wall and without activating the bumpers. These collisions are unlikely to harm the user or environment because the wheelchair's speed is low and the participants retain control of the wheelchair.

Type II collision: Collisions in which a cardboard tube was displaced by two to fourteen inches (Fig. 5c). These collisions may harm the environment but are unlikely to harm the user or the wheelchair.

Type III collision: Collisions in which a cardboard tube was displaced by more than fourteen inches (Fig. 5d) or fell over. Type III collisions may harm the user, the wheelchair or the environment in real world situations because the wheelchair's speed is high and participants do not have complete control of the wheelchair.

NASA-TLX: The NASA-TLX questionnaire is a selfreported, survey-based, validated, multidimensional rating procedure $[34,36,37]$. The NASA-TLX produces a total workload (TWL) score based on a weighted average of ratings on six subscales (Effort, Frustration, Performance, Mental Demand Physical Demand. and Temporal Demand). Each item is measured on a scale of $0-7$ where zero is the lowest possible score.

Mental Demand (TLX-MD) is defined as the mental and perceptual effort (e.g., thinking, deciding, calculating, remembering, searching, maneuvering) required to finish a navigation task. Physical Demand (TLXPD) is defined as the physical activity required (e.g., scanning, pushing, and pulling the cane, maneuvering the joystick, turning, controlling, activating) to finish the navigation task. Further, TLX-PD also represents whether the task was easy or demanding, slow or quick, restful or laborious. Temporal Demand (TLX-TD) is defined as the time pressure subjects felt due to the rate 


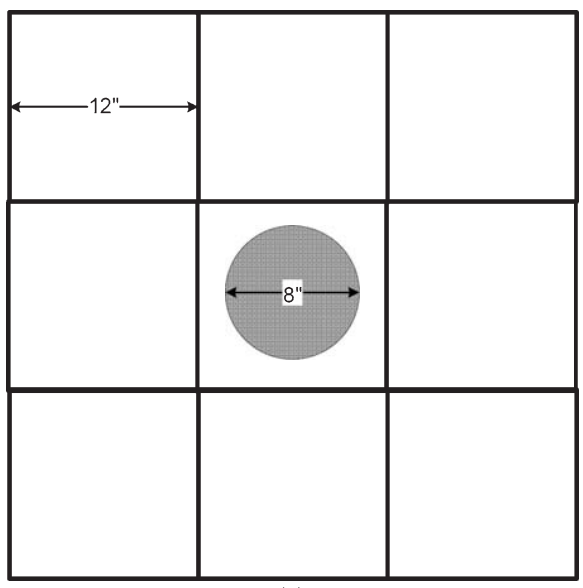

(a)

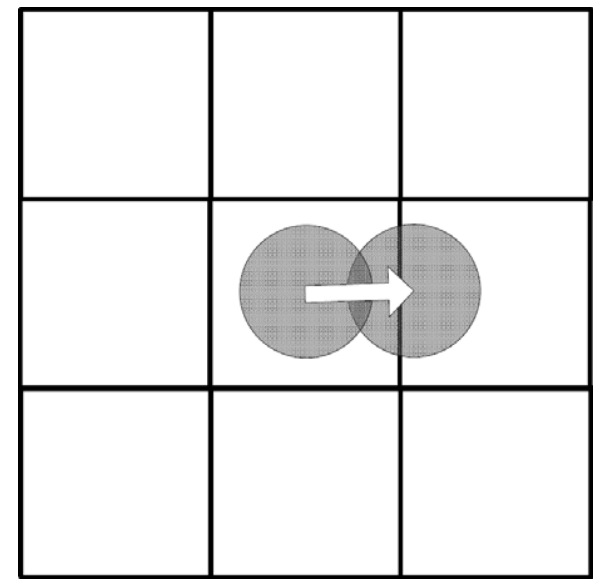

(c)

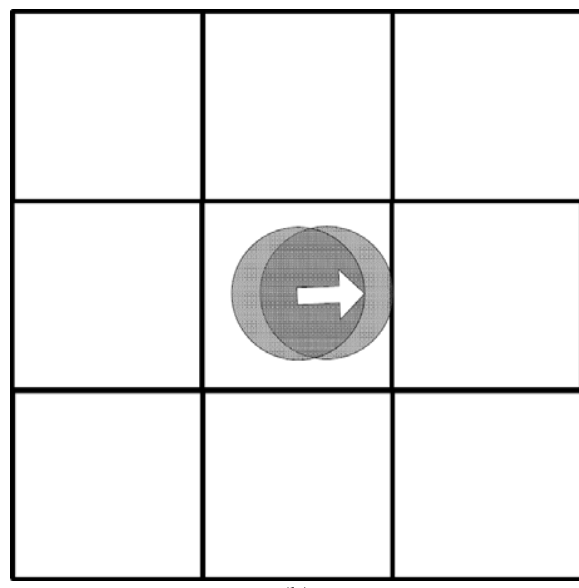

(b)

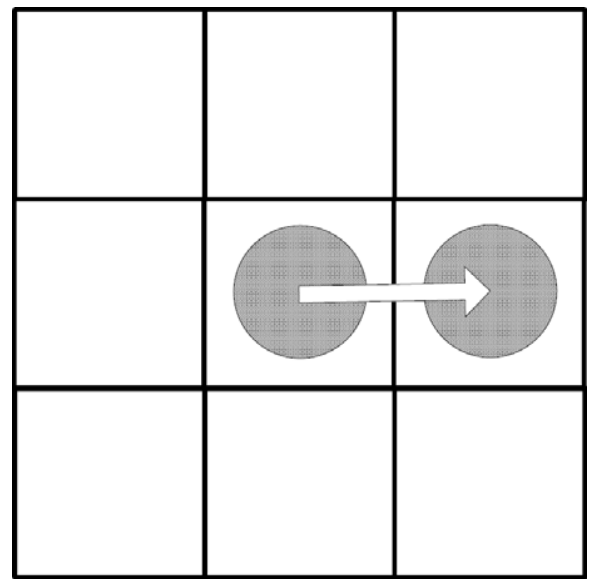

(d)

Fig. 5. (a) Obstacle position before collision. (b) Type I collision. (c) Type II collision. (d) Type III collision.

or pace at which the tasks or task elements occurred. TLX-TD also takes into account the pace of the task progression such as slow and leisurely or rapid and frantic. Perceived effort (TLX-E) is defined as the amount of work (both mental and physical) participants had to exert to achieve their level of performance. Effort and demand are different as demand is workload associated with the task and it is independent of users performance while effort represents the workload associated with interaction between the user and the task to achieve user's level of performance.

Frustration (TLX-F) measures the extent, to which a person feels insecure, discouraged, irritated, stressed and annoyed versus secure, gratified, content, relaxed and complacent during the task. Performance (TLX-P) is defined as how successful participants felt in accomplishing the goals of the task and how satisfied they were with their performance in accomplishing their goals. The participant's goal was to finish each trial within four minutes while maintaining safe navigation practices.

\subsection{Specific aims and hypotheses}

Safety of the wheelchair users and their surroundings is the key factor in prescription of powered mobility for people with disabilities. Testing of DSS's ability to avoid collisions and comparing it with a conventional navigation method (cane) will provide evidence for DSS's merits as an alternative navigation aid for people with visual and mobility impairments. We will use both quantitative as well as subjective measures to evaluate the performance of the DSS. 
The task completion time is a quantitative measure of DSS's performance, which can tell us whether users are able to complete the task using DSS within given time constraints. Comparing the task completion time using the DSS to using a cane will provide an understanding of the DSS's performance with respect to an established and frequently used clinical intervention. Task completion time and number of collisions are established measures of performance of the wheelchair navigation [38].

People with physical disabilities generally have limited physical stamina and reach. People with mobility and visual impairments currently use a cane or their hands to scan the environment for possible obstacles and environmental cues. This environment scanning could be physically challenging in many medical conditions for e.g. multiple sclerosis (MS), cerebral palsy (CP), arthritis, and chronic obstructive pulmonary disease (COPD). Evaluation and comparison of physical demand in a task with DSS and cane will give evidence about the merits of DSS in reducing the physical demand for people with mobility and visual impairments.

People with physical disabilities often have decline in cognitive abilities due to certain medical conditions e.g. aging, cerebral palsy, traumatic brain injury (TBI). Lower cognitive level might inhibit the learning to use the DSS if mental demand involved in operation is too much. Further, even if people have the cognitive abilities to learn to drive the DSS, constant high mental demand involved in operation of the DSS might keep users away from using it. However, the DSS is designed in a way such that it is intuitive and easy to learn, and therefore we predict that it will not impose an excessive demand on the cognitive resources of users.

If the DSS imposes an excessive physical and mental demand on users, it is likely that they will soon feel frustrated and not want to continue using the system. We therefore wanted to measure user frustration to get an idea of the extent to which the user feels insecure, discouraged, irritated, stressed, and annoyed versus secure, gratified, content, relaxed, and complacent during the task. We predict that users will feel less frustration while using the DSS compared to using a cane, because the obstacle-avoiding mechanism will give them a sense of security, and because it makes scanning backwards easier compared to using a cane.

Total workload will measure the performance of the DSS in six dimensions and combine them together to give a better understanding about the appropri- ateness of the technology to the user. Evaluation of TWL with DSS and comparison of it with cane will give an estimate of how difficult it is for the users to use the technology. Overall we hypothesize that when using the DSS participants will experience less total workload, primarily because of reduced physical demand, mental demands, effort, and lower level of frustration.

\subsection{Data analysis}

All analyses were performed using SPSS version 14.0. The Shapiro-Wilk test was used to check the normality of each dependent variable. If the Shapiro-Wilk statistic was greater than 0.01 , data was considered normally distributed. A General Linear Model (GLM) Repeated Measures ANOVA was used for analyses of normally distributed dependent variables with the significance level set at $p<0.05$. Pairwise comparisons were performed with a standard $t$-test with a Bonferroni adjustment for multiple comparisons. Data which were not normally distributed were analyzed using non-parametric tests for related samples. Friedman's test was used to compare the underlying distributions across all three experimental conditions with significance level set at $p<0.05$. Pair-wise comparisons between conditions were performed using the Wilcoxon Signed Ranks test with a significance level set at $p<0.05$.

\section{Results}

\subsection{Collisions}

Figure 6 shows the occurrence of collisions across three experimental conditions.

\subsubsection{Type I collisions}

Number of Type I Collisions per Trial (NCT-I) was greatest under the Cane condition with a mean of 2.26 $( \pm 1.85)$ per trial. The second largest NCT-I occurred under the Cane\&DSS condition, with a mean of 1.16 $( \pm 1.50)$. The lowest NCT-I occurred under the DSS condition, with a mean of $1.00( \pm 1.45)$. A significant difference existed among experimental conditions $\left(\chi^{2}[2,18]=8.035, p=0.018\right)$. Participants had significantly more NCT-I under the Cane condition than under the DSS condition $(Z=2.352, p=0.019)$ and the Cane\&DSS condition $(Z=2.278, p=0.023)$. There 


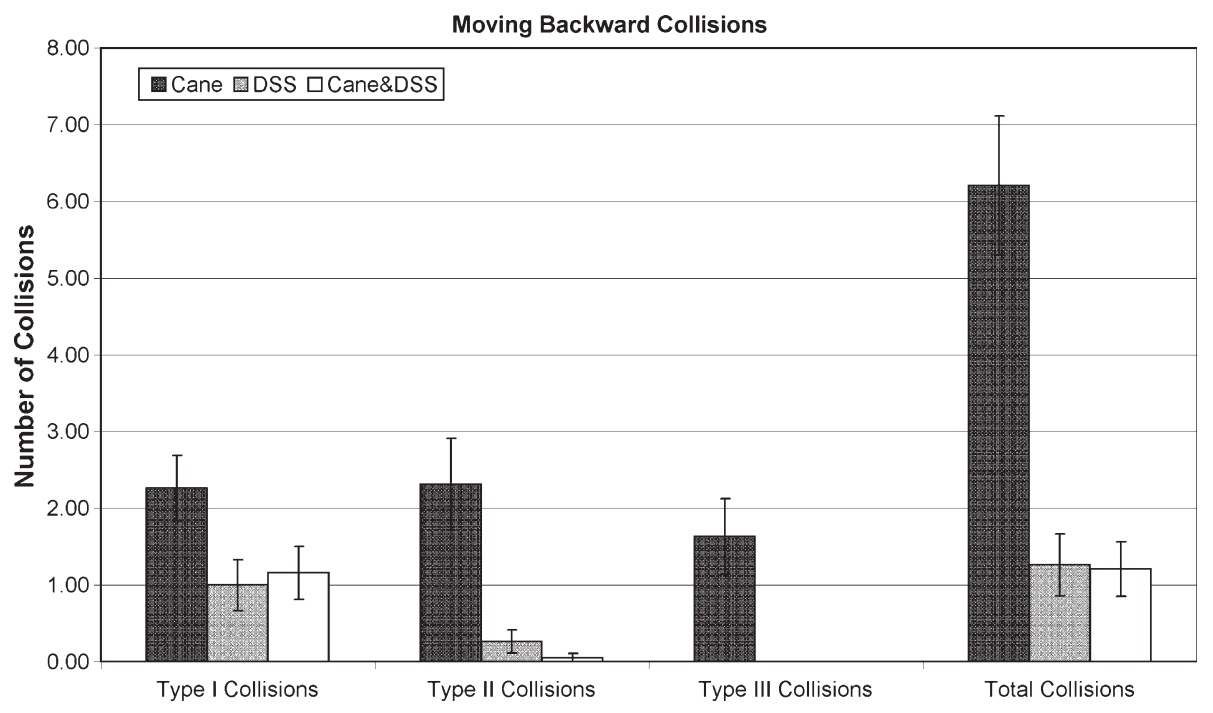

Fig. 6. Number of collisions.

was not a significant difference in NCT-I between the DSS and Cane\&DSS conditions $(Z=0.544, p=0.587)$.

\subsubsection{Type II collisions}

Number of Type II Collisions per Trial (NCT-II) was greatest under the Cane condition with a mean of 2.32 $( \pm 2.60)$ per trial. The second largest NCT-II occurred under the DSS condition with a mean of $0.26( \pm 0.65)$. The lowest NCT-II occurred under the Cane\&DSS condition with a mean of $0.05( \pm 0.23)$. A significant difference existed among driving conditions $\left(\chi^{2}(2\right.$, $N=19)=19.633, p=0.0001)$. Participants had significantly more NCT-II under the Cane condition than under the DSS condition $(Z=2.752, p=0.006)$ and the Cane\&DSS condition $(Z=3.329, p=0.001)$. There was not a significant difference in NCT-II between the DSS and Cane $\&$ DSS conditions $(Z=-1.30, p=0.194)$.

\subsubsection{Type III collisions}

Number of Type III Collisions per Trial (NCT-III) had a mean of $1.63( \pm 2.17)$ Type III Collisions under the Cane condition, but there were no Type III Collisions under either the DSS or Cane\&DSS conditions. A significant difference existed between conditions $\left(\chi^{2}(2, N=19)=22.00, p=0.0001\right)$. Participants had significantly more NCT-III under the Cane condition than under the Cane\&DSS condition $(Z=2.952, p=0.003)$ and the DSS condition $(Z=2.952, p=0.003)$.

\subsubsection{Total collisions}

The Cane condition had the greatest Total Number of Collisions per Trial (NCT-T) with a mean of 6.21 ( \pm 3.94$)$. The DSS condition had the second greatest NCT-T, with a mean of $1.26( \pm 1.76)$. The Cane\&DSS had the lowest NCT-T, with a mean of $1.21( \pm 1.55)$. A significant difference existed between conditions $\left(\chi^{2}(2, N=19)=21.848, p=0.0001\right)$. Participants had significantly greater NCT-T under the Cane condition than under the DSS condition $(Z=3.233$, $p=0.001)$ and the Cane\&DSS condition $(Z=3.525$, $p<0.0001)$. There was not a significant difference in NCT-T between the DSS and Cane\&DSS conditions $(Z=0.052, p=0.959)$.

\subsection{Task Completion Time}

Mean Task Completion Time (TCT) was lowest under the Cane condition at $93.75( \pm 32.20)$ seconds. Mean TCT was $96.81( \pm 27.46)$ seconds under the Cane\&DSS condition and was $98.41( \pm 46.36)$ seconds under the DSS condition. There was not a statistically significant difference between conditions $\left(\chi^{2}(2\right.$, 36) $=0.089, p=0.915)$.

\subsection{NASA-TLX}

Figure 7 shows the subjective workload ratings across experimental conditions. 


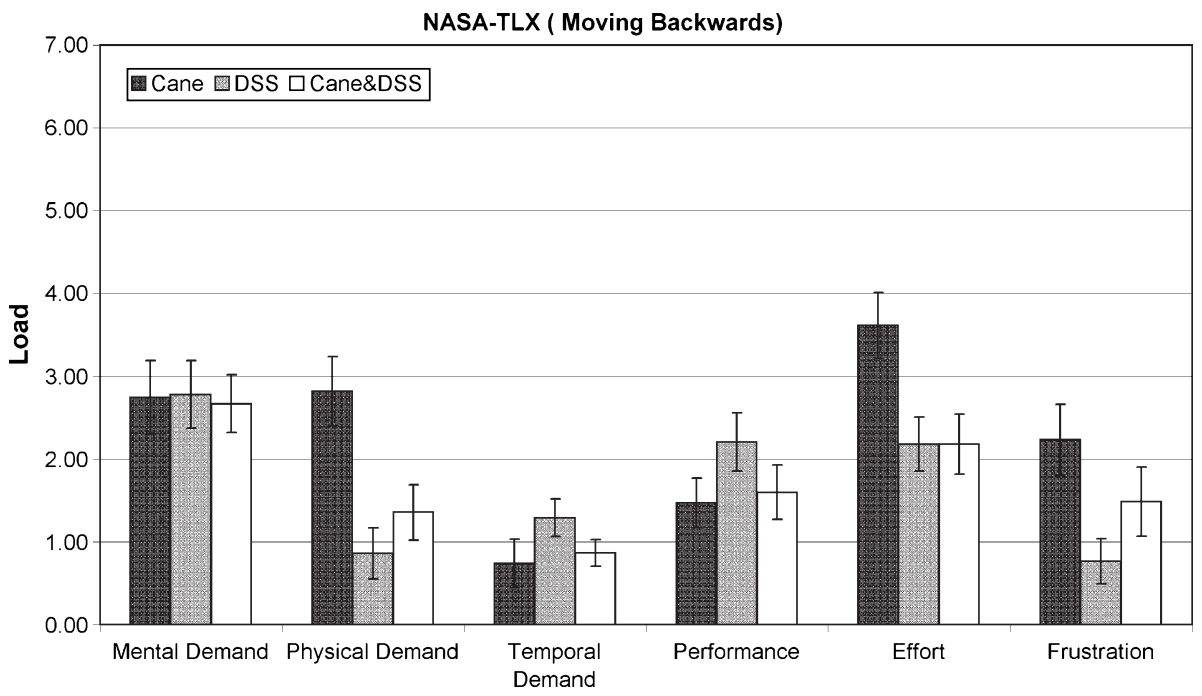

Fig. 7. NASA-TLX workload.

\subsubsection{Mental demand (TLX-MD)}

TLX-MD condition had a mean of $2.67( \pm 1.53)$ under the Cane\&DSS, a mean of $2.75( \pm 1.93)$ under the Cane condition and $2.78( \pm 1.79)$ under the DSS condition. There was not a significant difference between conditions $\left(\chi^{2}(2,36)=0.048, p=0.954\right)$.

\subsubsection{Physical demand (TLX-PD)}

TLX-PD had a mean of $0.86( \pm 1.35)$ under the DSS condition, a mean of $1.36( \pm 1.46)$ under the Cane\&DSS condition and a mean of $2.82( \pm 1.83)$ under the Cane condition. A significant difference existed between conditions $\left(\chi^{2}(2, N=19)=18.478\right.$, $p=0.0001)$. TLX-PD was significantly greater under the Cane condition than under the DSS condition $(Z=2.875, p=0.004)$ and the Cane\&DSS condition $(Z=2.770, p=0.006)$. There was not a significant difference in TLX-PD between the DSS and the Cane\&DSS conditions $(Z=1.633, p=0.102)$.

\subsubsection{Perceived effort (TLX-E)}

TLX-E had a mean of $2.18( \pm 1.42)$ under the DSS condition, a mean of $2.18( \pm 1.59)$ under the Cane\&DSS condition and a mean of $3.61( \pm 1.73)$ under the Cane condition. There was significant difference existed between conditions $\left(\chi^{2}(2\right.$, $N=19)=10.551, p=0.005)$. TLX-E was significantly greater under the Cane condition than under the DSS condition $(Z=2.702, p=0.007)$ and the Cane\&DSS condition $(Z=2.533, p=0.011)$. There was not a significant difference in TLX-E between the DSS condition and the Cane\&DSS condition $(Z=0.047$, $p=0.962$ ).

\subsubsection{Frustration (TLX-F)}

TLX-F had a mean of $0.76( \pm 1.19)$ under the DSS condition, a mean of $1.49( \pm 1.81)$ under the Cane\&DSS condition and a mean of $2.23( \pm 1.87)$ under the Cane condition. A significant difference existed between conditions $\left(\chi^{2}(2, N=19)=10.140\right.$, $p=0.006$ ). TLX-F was significantly higher under the Cane condition than under the DSS condition $(Z=3.067, p=0.002)$ and the Cane\&DSS condition $(Z=2.040, p=0.041)$. TLX-F was not significantly different under the Cane\&DSS and DSS conditions $(Z=1.687, p=0.092)$.

\subsubsection{Temporal demand (TLX-TD)}

TLX-TD had a mean of $0.74( \pm 1.29)$ under the Cane condition, a mean of $0.87( \pm 0.70)$ under the Cane\&DSS condition and a mean of $1.29( \pm 0.99)$ under the DSS condition. TLX-TD was not normally distributed under the Cane condition $(p=0.0001)$ but was normally distributed under the DSS and Cane\&DSS conditions (DSS: $p=0.057$, Cane\&DSS: $p=0.036)$. There was significant difference between conditions $\left(\chi^{2}(2, N=19)=10.941, p=0.004\right)$. TLXTD was significantly greater under the DSS condition than under the Cane condition $(Z=2.402, p=0.016)$ and the Cane\&DSS condition $(Z=2.432, p=0.015)$. 
There was no significant difference in TLX-TD between the Cane and the Cane\&DSS condition $(Z=0.828, p=0.408)$.

\subsubsection{Performance (TLX-P)}

TLX-P had a mean of $1.47( \pm 1.31)$ under the Cane condition, a mean of $1.60( \pm 1.44)$ under the Cane\&DSS condition and a mean of $2.21( \pm 1.54)$ under the DSS condition. TLX-P was normally distributed under the DSS condition $(p=0.148)$ but was not normally distributed under the Cane or Cane\&DSS conditions (Cane: $p=0.003$, DSS: $p=0.010$ ). There was a marginally significant difference between conditions $\left(\chi^{2}(2, N=19)=5.939, p=0.051\right)$. TLX-P was better under the Cane condition than under the DSS condition $(Z=2.002, p=0.056)$. There was no significant difference between the performance reported by the participants in the Cane and the Cane\&DSS condition $(Z=0.928, p=0.516)$. There was no significant difference between the performance reported by the participants in the DSS and the Cane\&DSS condition $(Z=1.201, p=0.218)$.

\subsubsection{Total workload (TLX-TWL)}

Total Workload (TLX-TWL) had a mean of 10.08 $( \pm 3.93)$ under the DSS condition, a mean of 10.16 $( \pm 4.46)$ under the Cane\&DSS condition and a mean of $13.62( \pm 4.76)$ under the Cane condition. There was significant difference in TLX-TWL across conditions $\left(\chi^{2}(2,36)=7.931, p=0.001\right)$. TLX-TWL was significantly higher under the Cane condition than under the DSS condition $(p=0.026)$ and the Cane\&DSS condition $(p=0.011)$. There was not a statistically significant between the DSS condition and the Cane\&DSS condition $(p=0.99)$.

\section{Discussion}

\subsection{Collisions}

In keeping with Hypothesis Q1, participants had significantly more collisions when using just cane alone than when using the DSS alone or DSS along with a cane. The DSS reduced the number and severity of collisions; nearly all collisions that occurred when the DSS was active were of the lowest severity (Type I). The more severe collisions (most Type II collisions and all Type III collisions) occurred when participants were using the cane alone. Type III collisions were pre- vented when using DSS because the sensors were able to slow or stop the chair before a collision occurred.

Most of the collisions when using the cane alone occurred primarily because participants could not devise a good strategy to scan the obstacles behind the wheelchair while driving backwards and were not able to detect the obstacles in time to stop the wheelchair. Second, participants could not maintain coordination between the wheelchair speed and the rate of obstacle scanning with the cane; even after detecting the obstacles, participants had difficulty bringing the wheelchair to a stop or changing direction in time to avoid a collision. Third, participants did not have an adequate understanding of the wheelchair's size and dynamics. Fourth, participants' scanning coverage was not wide enough to detect the obstacles in the corners of the wheelchair.

When using DSS alone or in combination with the cane, collisions occurred primarily because the sensor stop threshold was not appropriate in some sectors around the wheelchair. In addition, the DSS was unable to detect the obstacles in a "blind spot" behind the wheelchair. Most of the collisions that occurred when using the DSS were of very low severity (mainly Type I). The programmed sensor stop threshold was not appropriate when obstacles were present behind the wheelchair, allowing Type I collisions to occur when participants were turning and the rear bumper came in contact with the obstacles. All Type II collisions that occurred when using the DSS occurred because a certain area behind the wheelchair did not have adequate coverage from the sensors.

\subsection{Task Completion Time}

As hypothesized (Hypothesis Q2,) there was no difference in the task completion time in three experimental conditions. As shown in Fig. 8, TCT was slightly lower with the cane alone, but this performance was achieved at the expense of hitting significantly more obstacles. One reason for the lack of difference in TCT is that participants had difficulty scanning for obstacles behind the wheelchair using the cane, so they drove slowly to avoid the obstacles behind the wheelchair and made multiple stops. When using the DSS auditory feedback from the wheelchair did not enable the user to identify the exact position of obstacles around the wheelchair. In addition, Cross talk between sonar sensors occurs when transmitted ultrasound waves from one sensor are picked up by other 


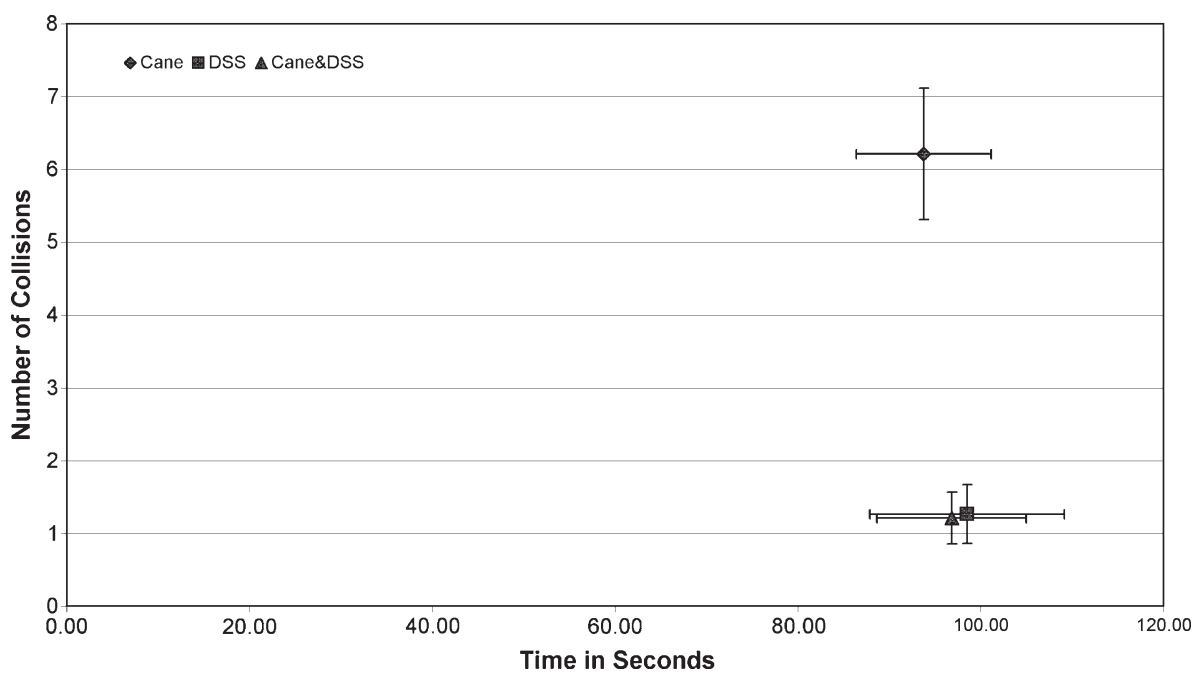

Fig. 8. Collisions vs. Time.

sensor and second sensor reports an incorrect range. The main cause of the cross talk is because all the URs operate at the same frequency $(42 \mathrm{KHz})$, therefore it is hard for sensors to distinguish if the reflected ultrasound wave is coming from their transmission or some other sensor's transmission. Sensor firing time, direction, and residual time ultrasound in air are the major causes of the cross-talk. In the DSS the even though the direction and timing of ranging of each UR is set in a way to avoid the cross talk, the high detection range of the MaxSonar-EZ series causes multiple reflections in few geographic situations and these reflected waves are picked up by other sensor and reported the false positive obstacles and wheelchair stopped itself.

This crosstalk between the URs made the wheelchair stop in instances where there were no obstacles, which caused participants to steer in the wrong direction and resulted in more time and effort. Finally, the DSS's inability to pass through narrow spaces when moving backwards made this navigation task difficult for the participants and added more time because they had to make multiple joystick manoeuvres.

\subsection{Physical demand}

As hypothesized, physical demand was lower when using the DSS alone or along with the cane. Participants felt additional physical demand when using the cane because they had to continuously turn themselves backwards and scan the area behind the wheelchair while driving the wheelchair with the joystick mounted in the front of the wheelchair. Physical demand was reduced when using the cane in combination with DSS since participants relied on the DSS for collision avoidance so they did not use the cane in this condition as much as they did in when using cane alone. Lowest physical demand was felt when participants were using the DSS alone; this demand was associated with the manoeuvring of the joystick.

Muscular weakness and/or neurological dysfunction are often accompanied by fatigue [39, 40]. Using a cane with a wheelchair may increase the severity of the fatigue which could interfere with activities of daily living (ADL). The reduced physical demand associated with use of the DSS can allow users to conserve their energy and use it for performing ADLs or social and occupational responsibilities.

\subsection{Mental demand}

As hypothesized there was no difference in the mental demand experienced across the experimental conditions. Able bodied participants never used the cane or the DSS before so they had to learn to use these devices for navigation assistance and that may be the reason they did not experience any difference in the mental demand.

A likely source of mental demand under all conditions was the need to construct and maintain a mental map of the test environment (the target, surrounding obstacles, the position and orientation of the wheelchair). Mental demand when using the cane 
alone resulted from the need to coordinate scanning and driving. This was difficult for participants because they had to use both their hands and most of the participants were not ambidextrous. Mental demand would also be expected to decrease over time as they learn to coordinate scanning and driving. Mental demand when using DSS was caused when participants had to estimate the position and sizes of obstacles based on auditory feedback from the wheelchair and then use this information to manoeuvre the wheelchair around obstacles and move towards the sound target. In addition, crosstalk between URs caused the wheelchair to act in ways that confused participants requiring them to work extra hard mentally to reach to the target.

\subsection{Frustration}

As hypothesized, participants reported far greater levels of frustration when using the cane alone. Using the cane caused frustration because participants felt insecure about hitting obstacles. Further, it was frustrating for the participants to sit and turn themselves backwards into an uncomfortable position to scan the area behind the wheelchair. The participants in this research relied on the auditory feedback to localize the position of the obstacles whenever the DSS stopped the movement of the wheelchair.

Many participants in the study had difficulty locating the position of the obstacles based on the auditory feedback from the DSS. Whenever the wheelchair is stopped by an obstacle, the DSS can provide auditory feedback from five sensor nodes (only one sensor node at a time) to show the position of the obstacles in any of five directions (front right, front left, right, left, back). Feedback pattern from the sensor nodes were kept similar to minimize the cognitive load on individuals. Participants had to rely on their sound localization skills to determine if the sound is coming from front left, front right, side left, side right, or from behind and these directions correspond to the position of obstacle which have triggered the stop of the DSS. Participants had difficulty determining which sensor node was providing the auditory feedback because of loudness of the sound generated by the end target. Secondly, the sound of the feedback was not loud enough, so it was hard for participants to hear the feedback. Good and intuitive auditory feedback can help drivers locate the obstacles and steer the wheelchair around them, without the need for multiple joystick manoeuvres. Auditory feedback from the DSS can be improved in by providing the distinct louder auditory feedback from each sensor node while not causing the excessive cognitive load. Auditory feedback is not always the most desirable choice for many people with disabilities primarily because family members and other people might not feel comfortable with the continuous beeping from the DSS in cluttered environments. Second, many people with disabilities don't want to show their disability in public, and auditory feedback may attract unwanted attention. Many participants suggested incorporating tactile feedback into the DSS. Tactile feedback will be more subtle and will not attract the attention of the public or family members but at the same time will be challenging to implement. Future version of the DSS should include tactile feedback so people with hearing impairments, which are common in the aging population, can benefit from the DSS.

Using a cane caused frustration because participants felt insecure about hitting obstacles and even after trying hard they could not stop the collisions when using the cane alone. Frustration with the DSS was mainly caused by the repeated manoeuvres required to steer the wheelchair around the obstacles, but these manoeuvres were decreased as participants learned to use the DSS. False positive stops because of the URs crosstalk perplexed participants and it caused frustration and confusion. It should be noted, however, that frustration was still low under both the conditions so it is unclear whether frustration was actually problematic.

\subsection{Perceived effort}

As hypothesized, effort when using the cane alone was significantly higher because participants had to put extra physical effort in scanning of the environment by cane. Further, there was mental effort involved in learning to coordinate both hands while navigating towards the target sound. Mental effort when using the cane is likely to decline as people learn to coordinate both hands (scanning and manoeuvring). Further, the physical effort component can be reduced by teaching efficient environment scanning strategies. While using the DSS it was the mental component which contributed mainly in the perceived effort because participants had difficulty in estimating the position of obstacles behind the wheelchair while relying only on the auditory feedback. This caused participants to make multiple joystick manoeuvres to move the 
wheelchair around an obstacle. Mental component in the perceived effort while using the DSS along with a cane was reduced because participants were able to use the cane to locate the position and the size of the obstacles and were able to reduce the number of joystick manoeuvres required to move the wheelchair around an obstacle.

\subsection{Temporal demand}

There was not a significant difference in temporal demand reported by the participants across three experimental conditions. Participants felt temporal demand because they were given four minutes to finish each trial and were given no feedback about the time elapsed during the trial. Temporal demands in all three conditions were low because all participants were able to finish the trials well within four minutes limit.

\subsection{Performance}

There was not a significant difference between conditions in participants' rating of their own performance. We relied on the performance evaluation based on the quantitative data from TCT and collisions. Participants had their best performance when using the DSS since they were able to reduce the number of collisions and at the same time able to finish the trial within four minutes.

\subsection{Total workload}

As hypothesized, total workload was significantly higher when using the cane alone. When using the cane alone, TLX-PD and TLX-E together were responsible for $47 \%$ of TLX-TWL. Further, TLX-MD accounted for $20 \%$ and TLX-F contributed $16 \%$ of TLX-TWL. Higher physical demand, perceived effort, and frustration with the cane alone resulted together in higher total workload with the cane alone in comparison to using the DSS alone or along with the cane.

\section{Conclusions}

The purpose of this research was to design and evaluate a clinically and commercially viable smart wheelchair architecture, which may facilitate independent mobility for individuals with a broad spectrum of disabilities. The main focus of this study was to determine if the DSS provides effective independent mobility to people with visual and mobility impairments especially when driving backwards. The results indicate that the DSS was able to provide reliable sensor coverage around the wheelchair and was able to avoid any catastrophic collisions which otherwise would have resulted in injury to the user or property damage. Nearly all hypotheses were supported by the data. The DSS reduced the number of collisions and the severity of collisions but did not increase the time required to complete navigation tasks. Participants reported experiencing less physical demand, less frustration and had to exert less effort in order to achieve better performance when using the DSS alone or along with a cane, compared to using a cane alone. The fact that all participants were young and able-bodied may have contributed to the relatively low workload reported for all three conditions.

The physical demand when using the cane alone was caused by the need for continuous scanning for obstacles. Non-ambulatory visually-impaired individuals with limited physical strength (e.g. aging, MS, $\mathrm{CP}$ ) might find the use of the cane extremely difficult because of the fatigue it produces. Physical demand when using the DSS was very low, and mainly caused by the continuous manoeuvring of the joystick. Another potential use of the DSS technology is training wheelchair navigation skills to the new or early stage wheelchair user. The DSS could also be useful for teaching early childhood powered mobility and joystick manipulation skills to children with disabilities.

In a survey of wheelchair users and health care professionals [38], the 30 powered wheelchair users rated collision avoidance as the most important factor in wheelchair manoeuvrability, while time to complete a task was ranked fourth. Thus, even though TCT was faster when using the cane alone, it is likely that using DSS for navigation will provide a more satisfying user experience due to the decrease in collisions.

Study reported in the present research was conducted in a controlled laboratory environment, which does not represent the real world scenarios that people with disabilities encounter in their day-to-day lives. Ultimately, the value of the DSS will be determined by its performance with the target population in the real world. Therefore, for higher ecological validity, the evaluation process should include "field trials" in which the DSS is used by target users for extended periods of time outside of the laboratory environment. One purpose of field trials is to compensate for the 
limitations of the lab trials, which must be conducted in a controlled environment. Another advantage of field trials is that users will be able to experience the DSS for several hours and will provide valuable feedback regarding the performance of the DSS in unconstrained environments. Investigators involved in the evaluation of the DSS in real world settings should also evaluate the users' ability to function when using the navigation assistance from the DSS using instruments such as Functioning Everyday With a Wheelchair (FEW) $[41,42]$ or Power Mobility Indoor/Community Driving Assessment (PIDA) [4, 43]. Further, anecdotal data obtained during the interviews will provide investigators with additional insight into specific situations that lead to system failures (collisions or software crashes), difficulties encountered when transporting the system, and problems positioning users within the chair.

When an individual obtains a new powered wheelchair, he or she often purchases the wheelchair through a clinician, who is responsible for configuring the wheelchair (e.g., input method, maximum velocity, and maximum acceleration), selecting seating and positioning hardware (e.g., cushions, lateral supports, and head rests), and mounting other equipment on the wheelchair (e.g., communication devices, lap trays, and ventilators). The assessment process typically involves reviewing the client's medical history, measuring the client's flexibility and range of motion, assessing the perceptual, motor, and cognitive skills required to operate a powered wheelchair, interviewing the client and caregivers, and, ultimately, some experimentation to identify a system that best meets the client's needs. Future evaluation of the DSS should involve occupational therapists, rehabilitation technologists and wheelchair suppliers who provide wheelchair seating and mobility services. These participants should complete mock powered mobility assessments involving the DSS to evaluate the system's ability to coexist with standard seating and positioning hardware. The trials will also provide insight into the training and documentation that clinicians will need to effectively utilize the DSS in practice. The mock powered mobility assessments will be used to evaluate the effectiveness of the instructional materials and configuration software of the DSS.

Reliable operation of the DSS in unknown environments requires it to detect upright obstacles as well as drop-offs. Drop-offs are responsible for most disastrous and fatal injuries among wheelchair users [13, $14,44]$. The present architecture of the DSS does not provide reliable solution for the detection of the drop-offs, which limits its operation to modified environments in which drop-offs have been eliminated. Future versions of the DSS should employ reliable drop-off detection technology and evaluate the user performance in environments which are not controlled.

Five foot high cylindrical tubes were used in this research as obstacles. The height and shape of these obstacles made them easy to be detected by sonar and IR sensors and this is likely to have enhanced the obstacle detection performance of the DSS. The next phase of trials should involve obstacles of varying height, shapes, colours, and surface textures, which will present varying level of detection difficulty for the proximity sensors.

The safety of the wheelchair driver and safety of the environment are key factors that clinicians and rehab practitioners consider when prescribing powered mobility for people with disabilities. The DSS's performance in avoiding collisions without significantly increasing task completion time presents it as an encouraging intervention for safe and independent mobility for visually impaired wheelchair users.

\section{Acknowledgements}

Tara Minkus assisted Dr. Sharma with data collection. This research was funded by the NIH (grant \#5R44HD040023-03) and the NSF (grant \#0540865).

\section{References}

[1] C. Butler, Effects of powered mobility on self-initiated behaviors of very young children with locomotor disability, Dev Med Child Neurol 28(3) (1986), 325-332. Available from http://www.ncbi.nlm.nih.gov/entrez/query.fcgi?cmd= Retrieve \&db=PubMed\&dopt=Citation \&list_uids $=2941328$.

[2] L. Rosenbloom, Consequences of impaired movement: a hypothesis and review, in: Movement and Child Development, London, England, 1975.

[3] B.A. Wright, Physical Disability - A Psychosocial Approach. Second edn, Harper \& Row: New York, 1983.

[4] K. Hall, J. Partnoy, S. Tenenbaum and D.R. Dawson, Power mobility driving training for seniors: a pilot study, Assist Technol 17(1) (2005), 47-56. Available from http:// www.ncbi.nlm.nih.gov/entrez/query.fcgi?cmd=Retrieve $\& \mathrm{db}$ $=$ PubMed\&dopt=Citation\&list_uids $=16121645$

[5] C. Miles-Tapping, Power wheelchairs and independent life styles, Canadian Journal of Rehabilitation 10 (1996), 137 145.

[6] R.C. Simpson, E.F. LoPresti and R.A. Cooper, How many people would benefit from a smart wheelchair? J Rehabil 
Res Dev 45(1) (2008), 53-71. Available from http://www. ncbi.nlm.nih.gov/entrez/query.fcgi?cmd $=$ Retrieve $\& d b=P u b$ Med\&dopt=Citation\&list_uids $=18566926$

[7] E. Trefler, S.G. Fitzgerald, D.A. Hobson, T. Bursick and R. Joseph, Outcomes of wheelchair systems intervention with residents of long-term care facilities, Assist Technol 16(1) (2004a), 18-27. Available from http://www.ncbi.nlm.nih. gov/entrez/query.fcgi?cmd=Retrieve $\& \mathrm{db}=$ PubMed $\& \mathrm{dopt}=$ Citation\&list_uids $=15357146$

[8] E. Trefler, S.G. Fitzgerald, D.A. Hobson, T. Bursick and R. Joseph, Outcomes of wheelchair systems intervention with residents of long-term care facilities, Assistive Technology 16(1) (2004b), 18-27. Available from http:// www.ncbi.nlm.nih.gov/entrez/query.fcgi?cmd=Retrieve $\& \mathrm{db}$ $=$ pubmed $\&$ dopt $=$ Abstract $\&$ list_uids $=15357146$

[9] A. Brandt, S. Iwarsson and A. Stahle, Older people's use of powered wheelchairs for activity and participation, J Rehabil Med 36(2) (2004), 70-77. Available from http:// www.ncbi.nlm.nih.gov/entrez/query.fcgi?cmd=Retrieve $\& \mathrm{db}$ $=$ PubMed\&dopt $=$ Citation\&list_uids $=15180221$

[10] M.L. Tolerico, D. Ding, R.A. Cooper, D.M. Spaeth, S.G. Fitzgerald, R. Cooper, A. Kelleher and M.L. Boninger, Assessing mobility characteristics and activity levels of manual wheelchair users, $J$ Rehabil Res Dev 44(4) (2007), 561-571. Available from http://www.ncbi. nlm.nih.gov/entrez/query.fcgi?cmd=Retrieve $\& \mathrm{db}=$ PubMed $\&$ dopt $=$ Citation $\&$ list_uids $=18247253$

[11] S. Guo, R.A. Cooper, T. Corfman, D. Ding and G. Grindle, Influence of wheelchair front caster wheel on reverse directional stability, Assist Technol 15(2) (2003), 98-104. Available from http://www.ncbi.nlm.nih.gov/entrez/ query.fcgi? $\mathrm{cmd}=$ Retrieve $\& \mathrm{db}=$ PubMed $\&$ dopt $=$ Citation $\&$ list _uids $=15137726$

[12] L. Fehr, W.E. Langbein and S.B. Skaar, Adequacy of power wheelchair control interfaces for persons with severe disabilities: a clinical survey, Journal of Rehabilitation Research and Development 37(3) (2000), 353-360.

[13] R.P. Gaal, N. Rebholtz, R.D. Hotchkiss and P.F. Pfaelzer, Wheelchair rider injuries: Causes and consequences for wheelchair design and selection, J Rehabil Res Dev 34(1) (1997), 58-71. Available from http://www.ncbi.nlm.nih. gov/entrez/query.fcgi?cmd=Retrieve $\& \mathrm{db}=$ PubMed $\& \mathrm{dopt}=$ Citation\&list_uids $=9021626$

[14] D. Gavin-Dreschnack, A. Nelson, S. Fitzgerald, J. Harrow, A. Sanchez-Anguiano, S. Ahmed and G. Powell-Cope, Wheelchair-related falls, Journal of Nursing Care Quality 20(2) (2005), 119-127. Available from http://search. ebscohost.com/login.aspx?direct=true $\& \mathrm{db}=\mathrm{hch} \& \mathrm{AN}=16428$ $624 \&$ site $=$ ehost-live

[15] T.A. Corfman, R.A. Cooper, S.G. Fitzgerald and R. Cooper, Tips and falls during electric-powered wheelchair driving: Effects of seatbelt use, legrests, and driving speed, Archives of Physical Medicine and Rehabilitation 84(12) (2003), 1797-1802. Available from http://www.sciencedirect.com/ science/article/B6WB6-4B5B35X-G/2/88772ac9df19f330d $152 \mathrm{db} 6 \mathrm{~d} 7 \mathrm{~b} 2 \mathrm{~d} 8 \mathrm{e} 5 \mathrm{f}$

[16] R. Simpson, E. LoPresti, S. Hayashi, S. Guo, D. Ding, W. Ammer, V. Sharma and R. Cooper, A prototype power assist wheelchair that provides for obstacle detection and avoidance for those with visual impairments, J Neuroeng Rehabil 2 (2005), 30. Available from http://www.ncbi.
nlm.nih.gov/entrez/query.fcgi?cmd=Retrieve $\& d b=$ PubMed $\&$ $\mathrm{dopt}=$ Citation\&list_uids $=16202136$

[17] M.P. LaPlante and H.S. Kaye, Demographics and trends in wheeled mobility equipment use and accessibility in the community, Assistive Technology 22(1) (2010), 3-17.

[18] W. Erickson and C. Lee, Disability Status Report: United States, Ithaca, NY, 2008.

[19] D. Ganoza, 11-15 March. Case study of a blind electric wheelchair driver, in: International Seating Symposium 11-15 March, 2009, Orlando.

[20] M.G. Greenbaum, S. Fernandes and S.F. Wainapel, Use of a motorized wheelchair in conjunction with a guide dog for the legally blind and physically disabled, Arch Phys Med Rehabil 79(2) (1998), 216-217. Available from http:// www.ncbi.nlm.nih.gov/entrez/query.fcgi?cmd=Retrieve $\& \mathrm{db}$ $=$ PubMed\&dopt=Citation\&list_uids $=9474006$

[21] Annonymous, Guiding blind people who are wheelchair users, in: Royal National Institute for the Blind, England, London (2002).

[22] M. Pranghofer, Wheels and White Canes: Tips for Helping Blind Wheelchair Users, 1996.

[23] R.C. Simpson, E.F. LoPresti, S. Hayashi, S. Guo, R. Frisch, A. Martin, W. Ammer, D. Ding and R.A. Cooper, The smart power assistance module for manual wheelchairs, in: Cambridge Workshop on Universal Access and Assistive Technology (CWUAAT), 22-24 March; Cambridge, UK: Cambridge University Press, (2004).

[24] R.C. Simpson, Smart wheelchairs: A literature review, J Rehabil Res Dev 42(4) (2005), 423-436. Available from http:// www.ncbi.nlm.nih.gov/entrez/query.fcgi?cmd=Retrieve $\& \mathrm{db}$ $=$ PubMed\&dopt=Citation\&list_uids $=16320139$

[25] D. Kelly, The enhancement of mobility for individuals who are both physically and visually disabled, in Proceedings of the RESNA'99 Annual Conference Long Beach, CA, 1999, pp. 227-229.

[26] P.D. Nisbet, J. Craig, J.P. Odor and S. Aitken, 'Smart' wheelchairs for mobility training, Technology and Disability 5 (1996), 49-62.

[27] R.C. Simpson, D. Poirot and F. Baxter, The hephaestus smart wheelchair system, IEEE Trans Neural Syst Rehabil Eng 10(2) (2002), 118-122. Available from http://www. ncbi.nlm.nih.gov/entrez/query.fcgi?cmd=Retrieve $\& d b=P u b$ Med\&dopt=Citation\&list_uids $=12236449$

[28] R. Simpson, E. Lopresti, S. Hayashi, I. Nourbakhsh and D. Miller, The smart wheelchair component system, J Rehabil Res Dev 41(3B) (2004), 429-442. Available from http:// www.ncbi.nlm.nih.gov/entrez/query.fcgi?cmd=Retrieve $\& \mathrm{db}$ $=$ PubMed\&dopt $=$ Citation\&list_uids $=15543461$

[29] E. Demeester, A. Huntemann, D. Vanhooydonck, G. Vanacker, H. Van Brussel and M. Nuttin, User-adapted plan recognition and user-adapted shared control: A Bayesian approach to semi-autonomous wheelchair driving, Autonomous Robots 24(2) (2008), 193-211.

[30] S.P. Levine, D.A. Bell, L.A. Jaros, R.C. Simpson, Y. Koren and J. Borenstein, The NavChair assistive wheelchair navigation system, IEEE Trans Rehabil Eng 7(4) (1999), 443-451. Available from http://www.ncbi. nlm.nih.gov/entrez/query.fcgi?cmd=Retrieve $\& d b=$ PubMed $\&$ dopt $=$ Citation $\&$ list_uids $=10609632$

[31] E. LoPresti, V. Sharma, R. Simpson and C. Mostowy, Performance testing of a collision avoidance system for power 
wheelchairs, Journal of Rehabiliation Research and Development (2010), Under Review.

[32] V. Sharma, R. Simpson, E. LoPresti and M. Schmeler, Evaluation of a semi-autonomous navigation assistance system for power wheelchairs with blindfolded able bodied individuals, Journal of Rehabiliation Research and Development (2010), Under Review.

[33] R.A. Cooper, T. Thorman, R. Cooper, M.J. Dvorznak, S.G. Fitzgerald, W. Ammer, G. Song-Feng and M.L. Boninger, Driving characteristics of electric powered wheelchair users: How far, fast and often do people drive? Archives of Physical Medicine and Rehabilitation 83(2) (2002), 250-255. Available from http://www2.archives-pmr.org/ scripts/om.dll/serve?action=searchDB \&searchDBfor=art\& artType $=$ abs\&id $=$ aapmr0830250\&nav $=$ abs

[34] S.G. Hart, NASA-Task Load Index (NASA-TLX); 20 years later, Proceedings of the Human Factors and Ergonomics Society 50th Annual Meeting, HFES, Santa Monica, 2006, pp. 904-908.

[35] S.G. Hart and L.E. Staveland, Development of NASA-TLX (Task Load Index): Results of empirical and theoretical research, in: Human Mental Workload, P.A. Hancock and N. Meshkati, eds, North Holland Press, Amsterdam, 1988.

[36] J.M. Noyes and D.P. Bruneau, A self-analysis of the NASATLX workload measure, Ergonomics 50(4) (2007), 514-519. Available from http://www.ncbi.nlm.nih.gov/entrez/query. fcgi? $\mathrm{cmd}=$ Retrieve $\& \mathrm{db}=$ PubMed $\&$ dopt=Citation\&list_uids= 17575712

[37] Y.M. Xiao, Z.M. Wang, M.Z. Wang and Y.J. Lan, The appraisal of reliability and validity of subjective workload assessment technique and NASA-task load index, Zhonghua Lao Dong Wei Sheng Zhi Ye Bing Za Zhi 23(3) (2005), 178-181. Available from http://www.ncbi.nlm.nih. gov/entrez/query.fcgi?cmd=Retrieve $\& \mathrm{db}=$ PubMed\&dopt= Citation\&list_uids $=16124892$

[38] P.J. Holliday, A. Mihailidis, R. Rolfson and G. Fernie, Understanding and measuring powered wheelchair mobility and manoeuvrability, Part I. Reach in confined spaces, Disabil
Rehabil 27(16) (2005), 939-949. Available from http:// www.ncbi.nlm.nih.gov/entrez/query.fcgi? $\mathrm{cmd}=$ Retrieve \&db $=$ PubMed\&dopt=Citation\&list_uids $=16096247$

[39] L. Krupp, Fatigue is intrinsic to multiple sclerosis (MS) and is the most commonly reported symptom of the disease, Mult Scler 12(4) (2006), 367-368. Available from http:// www.ncbi.nlm.nih.gov/entrez/query.fcgi? $\mathrm{cmd}=$ Retrieve $\& \mathrm{db}$ $=$ PubMed\&dopt $=$ Citation\&list_uids $=16900749$

[40] L.B. Krupp, L.A. Alvarez, N.G. LaRocca and L.C. Scheinberg, Fatigue in multiple sclerosis, Arch Neurol 45(4) (1988), 435-437. Available from http://www.ncbi.nlm.nih gov/entrez/query.fcgi?cmd=Retrieve $\& \mathrm{db}=$ PubMed\&dopt= Citation\&list_uids $=3355400$

[41] T. Mills, M.B. Holm, E. Trefler, M. Schmeler, S. Fitzgerald and M. Boninger, Development and consumer validation of the Functional Evaluation in a Wheelchair (FEW) instrument, Disabil Rehabil 24(1-3) (2002), 38-46. Available from http://www.ncbi.nlm.nih.gov/entrez/query.fcgi? $\mathrm{cmd}=$ Retrieve $\& d b=$ PubMed $\&$ dopt $=$ Citation\&list_uids $=11827153$

[42] T.L. Mills, M.B. Holm and M. Schmeler, Test-retest reliability and cross validation of the functioning everyday with a wheelchair instrument, Assist Technol 19(2) (2007), 61-77. Available from http://www.ncbi.nlm.nih.gov/entrez/ query.fcgi? $\mathrm{cmd}=$ Retrieve $\& \mathrm{db}=$ PubMed \&dopt=Citation\&list _uids $=17727074$

[43] L. Letts, D. Dawson, I. Bretholz, E. Kaiserman-Goldenstein, J. Gleason, E. McLellan, L. Norton and C. Roth, Reliability and validity of the power-mobility community driving assessment, Assist Technol 19(3) (2007), 154-163; quiz 127. Available from http://www.ncbi.nlm.nih.gov/entrez/ query.fcgi? $\mathrm{cmd}=$ Retrieve $\& \mathrm{db}=\mathrm{PubMed} \& \mathrm{dopt}=$ Citation $\&$ list _uids $=17937057$

[44] S. Marshall, Wheelchair rider injuries: Causes and consequences for wheelchair design and selection, $J$ Rehabil Res Dev 34(2) (1997), vi. Available from http://www.ncbi. nlm.nih.gov/entrez/query.fcgi? $\mathrm{cmd}=$ Retrieve $\& \mathrm{db}=$ PubMed $\&$ dopt $=$ Citation\&list_uids $=9108340$ 

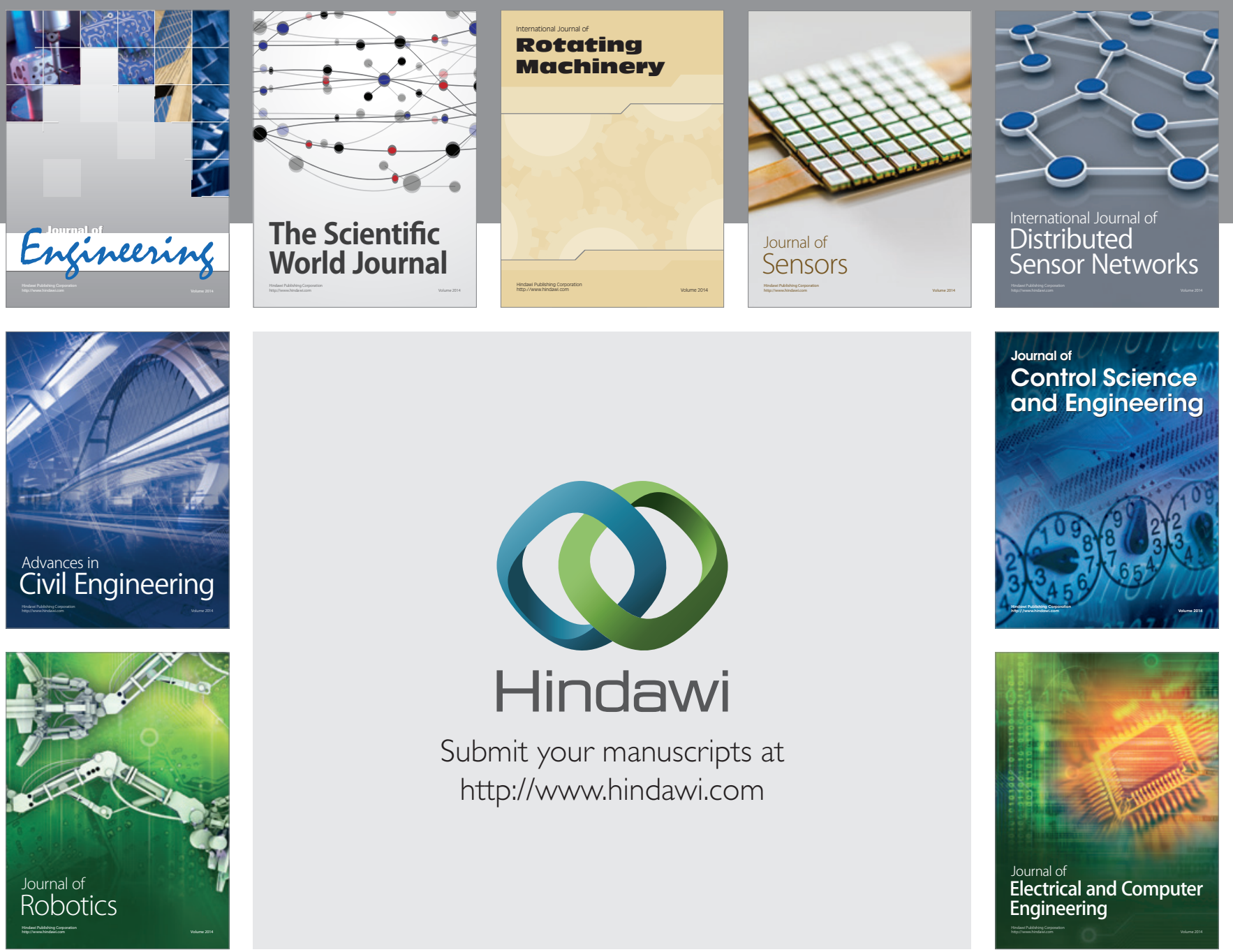

Submit your manuscripts at

http://www.hindawi.com
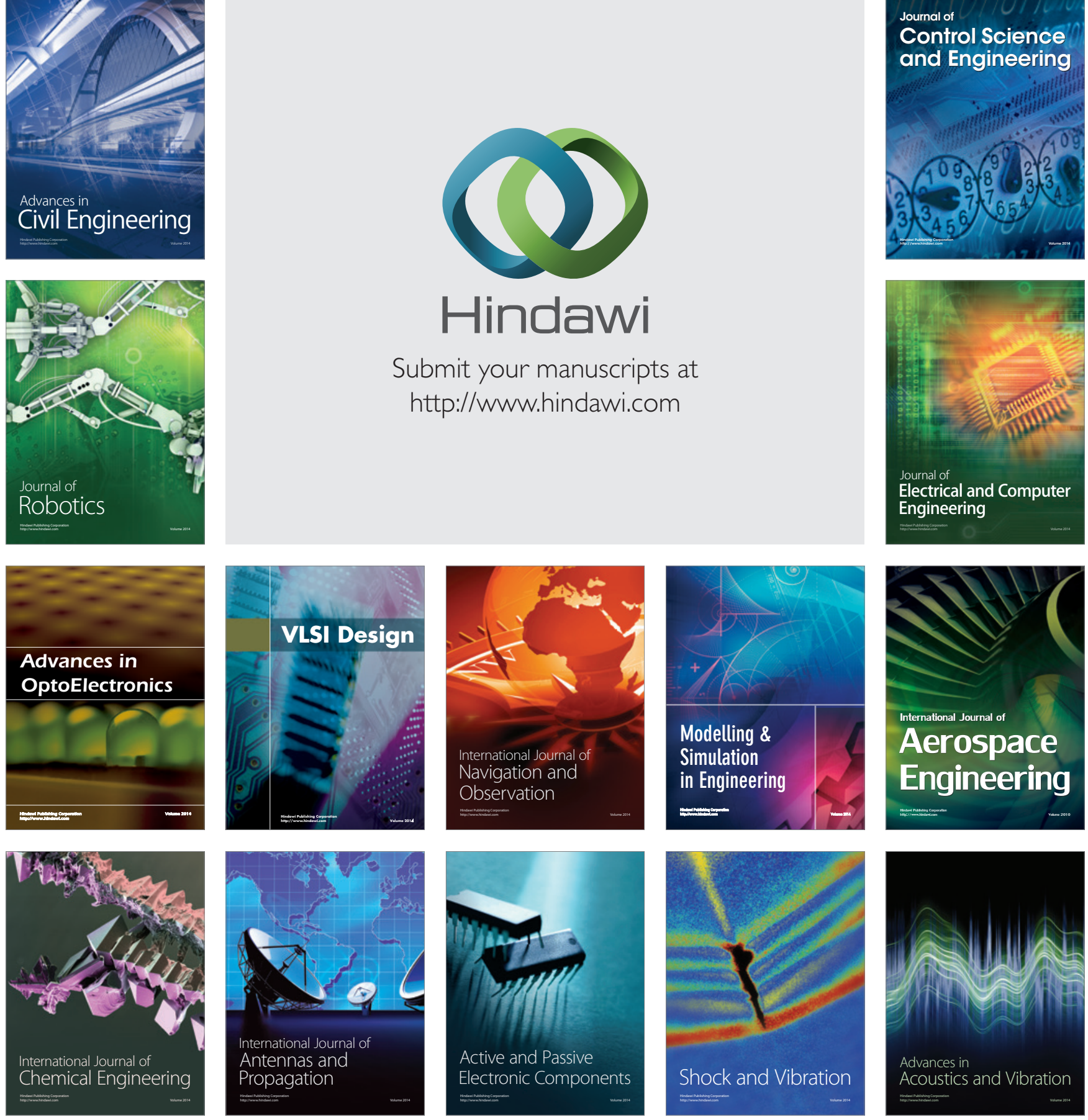